\title{
EFFICIENT WALD TESTS FOR FRACTIONAL UNIT ROOTS
}

\author{
By IGNACIO N. LOBATO AND CARLOS VELASCO ${ }^{1}$
}

\begin{abstract}
In this article we introduce efficient Wald tests for testing the null hypothesis of the unit root against the alternative of the fractional unit root. In a local alternative framework, the proposed tests are locally asymptotically equivalent to the optimal Robinson Lagrange multiplier tests. Our results contrast with the tests for fractional unit roots, introduced by Dolado, Gonzalo, and Mayoral, which are inefficient. In the presence of short range serial correlation, we propose a simple and efficient two-step test that avoids the estimation of a nonlinear regression model. In addition, the first-order asymptotic properties of the proposed tests are not affected by the preestimation of short or long memory parameters.
\end{abstract}

KEYWORDS: Long memory, serial correlation, Dickey-Fuller test, Lagrange multiplier test, fractional processes, local power.

\section{INTRODUCTION}

TESTING FOR NONSTATIONARITY of a time series is routinely performed as a first step in econometric modeling. For instance, in the traditional $I(0) / I(1)$ framework, unit-root tests have been applied frequently. Recently, there has been considerable interest in studying long memory series, where the degree of nonstationarity is characterized by a fractional integration parameter that takes values in a continuum. Analysis with long memory series has posed new problems and led to the development of new asymptotic and optimality theory. For instance, Robinson (1991, 1994a) proposed Lagrange multiplier (LM) tests in both the frequency and the time domain, and Dolado, Gonzalo, and Mayoral (2002; hereafter DGM) introduced a test based on an auxiliary regression for the null of the unit root against the alternative of fractional integration.

In the basic framework, $y_{t}$ denotes a fractionally integrated process whose true order of integration is $d$, denoted as $I(d)$,

$$
\Delta^{d} y_{t} 1\{t>0\}=\varepsilon_{t}
$$

$$
(t=1,2, \ldots),
$$

where $\varepsilon_{t}$ are independent and identically distributed (i.i.d.) random variables with zero mean and finite variance, and $1\{\cdot\}$ denotes the indicator function.

\footnotetext{
${ }^{1}$ We thank a co-editor and two referees for very useful comments, J. Arteche, M. Avarucci, M. Delgado, J. Dolado, L. Gil-Alaña, J. Gonzalo, J. Hidalgo, L. Mayoral, P. Perron, W. Ploberger, and P. Robinson for useful conversations, and seminar participants at the London School of Economics and at the 2005 Econometric Society World Congress. This research was financed by the Spanish Ministerio de Educación y Ciencia (SEJ2004-04583/ECON). Part of this research was carried out while Lobato was vis ting Universidad Carlos III de Madrid thanks to the Spanish Secretaría de Estado de Universidades e Investigación (SAB2004-0034). Lobato acknowledges financial support from Asociación Mexicana de Cultura and from the Mexican Consejo Nacional de Ciencia y Tecnología (CONACYT) under project grant 41893-S.
} 
The fractional difference operator $\Delta^{d}=(1-L)^{d}$ is defined in terms of the lag operator $L$ by the formal expansion

$$
\Delta^{\alpha}:=\sum_{i=0}^{\infty} \pi_{i}(\alpha) L^{i}
$$

for any real $\alpha$, where for $\alpha \neq 1,2, \ldots$,

$$
\pi_{i}(\alpha)=\frac{\Gamma(i-\alpha)}{\Gamma(i+1) \Gamma(-\alpha)},
$$

and $\Gamma$ is the Gamma function, with $\Gamma(0) / \Gamma(0)=1$, so the first coefficients are $\pi_{0}(\alpha)=1$ and $\pi_{1}(\alpha)=-\alpha$. From now on, in the notation we will suppress the truncation in (1) for nonpositive $t$, assuming implicitly that $y_{t}=\varepsilon_{t}=0, t \leq 0$.

We consider testing the null hypothesis

$$
H_{0}: d=1
$$

versus either a simple alternative

$$
H_{A}: d=d_{A}<1
$$

or a composite alternative

$$
H_{1}: d<1 .
$$

Dolado, Gonzalo, and Mayoral (2002) proposed to test the null hypothesis by means of the $t$ statistic of the coefficient of $\Delta^{d_{1}} y_{t-1}$ in the ordinary least squares (OLS) regression

$$
\Delta y_{t}=\phi_{1} \Delta^{d_{1}} y_{t-1}+u_{t}
$$$$
(t=1, \ldots, T),
$$

where $T$ denotes the sample size. They called this $t$ ratio the fractional DickeyFuller test, based on a particular analogy with the Dickey and Fuller (1979) test that led them to interpret $d_{1}$ as "the true value of $d$ under the alternative hypothesis"; hence, they proposed using $d_{1}=d_{A}$ when testing against $H_{A}$ and using a consistent estimator of $d$ when testing against $H_{1}$.

Notice that the null and alternative hypotheses can be expressed in model (2) in terms of $\phi_{1}$, which is defined as the probability limit of the OLS coefficient of $\Delta^{d_{1}} y_{t-1}$. Under $H_{0}, \phi_{1}=0$ because $\Delta y_{t}$ is white noise and, hence, is uncorrelated with $\Delta^{d_{1}} y_{t-1}$ for any value of $d_{1}$. By contrast, under the alternative, using $\Delta y_{t}=\Delta^{1-d} \varepsilon_{t}=\varepsilon_{t}+(d-1) \varepsilon_{t-1}+\cdots$ and $\Delta^{d_{1}} y_{t-1}=\Delta^{d} y_{t-1}=\varepsilon_{t-1}$ when $d_{1}=d$ is employed, it is simple to show that $\phi_{1}=d-1<0$. Because $\phi_{1}$ is also negative for any $d_{1}>0.5$, the regression model (2) can be used to test the null hypothesis by checking the significance of the regressor $\Delta^{d_{1}} y_{t-1}$ with a one-sided $t$-ratio test. 
However, note that the null hypothesis could also be tested by testing the significance of alternative regressors. In fact, given that $\Delta y_{t}$ is i.i.d. under the null, $\Delta^{d_{1}} y_{t-1}$ could be replaced in (2) by any function of the past, and the associated coefficient would still be zero, whereas, under the alternative, this coefficient would be negative for any function of the past with negative covariance with $\Delta y_{t}$.

This article questions the use of the regressor $\Delta^{d_{1}} y_{t-1}$ proposed by DGM and examines carefully the optimal selection of the regressor in a regression model like (2) to conduct inference on the degree of integration of $y_{t}$. We argue that $\Delta^{d_{1}} y_{t-1}$ is not the best class of regressors one can choose. To grasp the intuition behind this argument, consider all the regressors that lead to a test statistic whose asymptotic null distribution is the standard normal (for instance, $\Delta^{d_{1}} y_{t-1}$ with $\left.d_{1}>0.5\right)$. Note that the test that maximizes the power among this group maximizes the correlation between the regressand and the regressor, and thus it is based on a regression model where the errors are serially uncorrelated and uncorrelated to the regressor. Therefore, a regressor such as $\Delta^{d_{1}} y_{t-1}$ cannot be optimal because, under the alternative hypothesis, there exist no values of $\phi_{1}$ and $d_{1}$ that guarantee that the error term $u_{t}$ in model (2) is serially uncorrelated and orthogonal with the regressor $\Delta^{d_{1}} y_{t-1}$. In this sense, model (2) is misspecified because it does not include the data generating process (DGP) defined by (1) as a particular case under the alternative hypothesis. In particular, the errors of the model, $u_{t}$, are different from the innovations of the process, $\varepsilon_{t}$, defined in (1). This misspecification implies that OLS estimation and the resulting $t$ test based on regression (2) are inefficient, even when $d_{1}$ is optimally chosen, see Lobato and Velasco (2006) for optimal selections for $d_{1}$.

In this article we propose the use of an alternative regression model based on (1), which leads to an efficient $t$ test that can also be interpreted as a Wald test because the relevant slope coefficient in the estimated regression is linearly related to the parameter of interest. The proposed Wald test is asymptotically efficient against local alternatives because it is asymptotically equivalent to Robinson's $(1991,1994 a)$ LM test, which is optimal in a Gaussian framework. In particular, we show that our $t$ test statistic is locally asymptotically equivalent to Robinson's time-domain LM test statistic

$$
L M=T^{1 / 2}\left(\frac{\pi^{2}}{6}\right)^{-1 / 2} \sum_{j=1}^{T-1} j^{-1} \widehat{\rho}_{\Delta y}(j),
$$

where $\widehat{\rho}_{\Delta y}(j)$ denotes the sample autocorrelation of order $j$ of $\Delta y_{t}$. This statistic has also been attributed to Tanaka (1999), but note that it previously appeared in Robinson (1991); see also Robinson (1994b).

The plan of the article is the following. Section 2 proposes and analyzes the new efficient fractional regression test. Section 3 studies the consequences of allowing for serial correlation in $\varepsilon_{t}$ in (1), and proposes a simple and efficient two-step test. Section 4 reports a Monte Carlo exercise on the finite sample 
performance of the considered tests. Section 5 concludes and proposes some lines of further research.

\section{AN OPTIMAL WALD TEST}

In this section we study carefully the optimal selection of the regressor and develop an efficient Wald type test. To motivate the selection of the proposed regressor, note that for any $d$ we can rewrite the DGP (1) as

$$
\Delta y_{t}=\left(\Delta-\Delta^{d}\right) y_{t}+\varepsilon_{t}=\left(1-\Delta^{d-1}\right) \Delta y_{t}+\varepsilon_{t},
$$

where the error term $\varepsilon_{t}$ is truly i.i.d. under (1), both under the null and under the alternative hypotheses, and where the variable $\left(1-\Delta^{d-1}\right) \Delta y_{t}$ does not contain $\Delta y_{t}$ because

$$
\left(1-\Delta^{d-1}\right) \Delta y_{t}=(d-1) \Delta y_{t-1}+\sum_{j=2}^{t-1} \pi_{j}(d-1) \Delta y_{t-j} .
$$

Equation (4) can also be written as

$$
\Delta y_{t}=\varphi_{2}\left(\Delta^{d-1}-1\right) \Delta y_{t}+\varepsilon_{t} \quad(t=1, \ldots, T),
$$

where $\varphi_{2}=0$ under the null and $\varphi_{2}=-1$ under the alternative. Equation (6) suggests the use of the regressor $\left(\Delta^{d_{2}-1}-1\right) \Delta y_{t}$, where $d_{2}$ denotes the input of the new test to distinguish it from the input $d_{1}$ in DGM's test. However, note that $d_{2}$ cannot take the value $d_{2}=1$, which would make the regressor equal to zero, as (5) indicates. In addition, note that a one-sided $t$-ratio test statistic for the significance of $\varphi_{2}$ in (6) should not be used with values of $d_{2}>1$, because the sign of the coefficients of $\left(\Delta^{d_{2}-1}-1\right) \Delta y_{t}$ in the expansion (5) changes depending on whether $d_{2}-1$ is positive or negative. Therefore, to make the regressor continuous at $d_{2}=1$, instead of (6), we propose to employ the rescaled regression model

$$
\Delta y_{t}=\phi_{2} z_{t-1}\left(d_{2}\right)+u_{t}
$$

$$
(t=1, \ldots, T)
$$

where

$$
z_{t-1}\left(d_{2}\right)=\frac{\left(\Delta^{d_{2}-1}-1\right)}{1-d_{2}} \Delta y_{t} .
$$

We propose to test the null hypothesis by testing the significance of the coefficient of $z_{t-1}\left(d_{2}\right)$, with $d_{2}>0.5$, in (7) by means of a left-sided test based on the $t$-ratio test statistic, denoted by $t_{\phi}$.

Note that when $d_{2}=d$ in (7), the true value of $\phi_{2}$ is obtained immediately by $\phi_{2}=\varphi_{2}(1-d)=d-1$, which maps the hypotheses on the parameter $d$ 
continuously into $\phi_{2}$. That is, under the null, $\phi_{2}=\varphi_{2}=0$, and under the alternative, $\phi_{2}$ takes negative values that are larger in absolute value the further $d$ is from the null. Note the analogy with the original Dickey-Fuller test based on model $\Delta y_{t}=\phi y_{t-1}+u_{t}$, where $\phi=\rho-1$ and $\rho$ denotes the first-order autocorrelation. In this case $\phi=0$ (or $\rho=1)$ is the null and $\phi<0($ or $\rho<1)$ is the alternative. Both tests are Wald because of the direct relationship between the slope coefficient in the auxiliary regression and the parameter of interest.

The model (7) is obviously related to the DGP (4) as we analyze next. Under the null hypothesis, $\Delta y_{t}$ is i.i.d. and so $\phi_{2}=0$ for any value of $d_{2}$, and model (7) is properly specified with $u_{t}=\varepsilon_{t}$. Under the alternative hypothesis, when $d_{2}$ is chosen equal to $d, \phi_{2}=d-1$ and model (7) is again properly specified with $u_{t}=\varepsilon_{t}$. However, when $d_{2}$ is chosen differently from the true value of $d$, this property is lost because the errors $u_{t}$ are not i.i.d. and, in consequence, $\phi_{2}$ (defined as the probability limit of the OLS estimator of the coefficient of $\left.z_{t-1}\left(d_{2}\right)\right)$ is no longer $d-1$. Therefore, under the alternative, to maximize the correlation between the regressand and the regressor (and hence, to maximize the power of the corresponding $t$ test), the researcher should set $d_{2}=d$. Other selections of $d_{2}$ would render consistent but inefficient tests compared to the selection $d_{2}=d$.

Comparing models (2) and (7), we see that the only difference with DGM's test is the use of the regressor $z_{t-1}\left(d_{2}\right)$ instead of the regressor $\Delta^{d_{1}} y_{t-1}$. Both regressors can be expressed as a linear combination of past values of $y_{t}$, and if we denote by $c_{j}^{z}$ and $c_{j}^{o}$ the coefficients of $y_{t-j}$ for $z_{t-1}(d)$ and $\Delta^{d} y_{t-1}$, respectively, it is simple to see that $c_{j}^{z}=(d-1) c_{j+1}^{o}$ for $j \geq 2$. However, the use of regressor $z_{t-1}\left(d_{2}\right)$ instead of $\Delta^{d_{1}} y_{t-1}$ leads to an important difference. Whereas for model (7) there exists a value of the pair $\left(d_{2}, \phi_{2}\right)$, namely $\left(d_{2}, \phi_{2}\right)=(d, d-1)$, that leads to errors which are i.i.d. and independent of the regressor under the alternative hypothesis, for model (2) there exists no value of the pair $\left(d_{1}, \phi_{1}\right)$ with that property. Therefore, the $t$ test based on the OLS estimation of (2) is inefficient (for any selection of $d_{1}$ ) compared to the $t$ test based on the OLS estimation of (7) that uses $d_{2}=d$. The intuition behind this inefficiency is straightforward: the regressor $z_{t-1}(d)$ contains all relevant past information to forecast $\Delta y_{t}$, whereas $\Delta^{d_{1}} y_{t-1}$ does not, irrespective of the value of $d_{1}$.

In addition, note that in the $d_{2}=1$ case, the indetermination $0 / 0$ in (8) is solved using l'Hôpital rule, because, as $d_{2} \rightarrow 1$, the ratio $\left(\Delta^{d_{2}-1}-1\right) /\left(1-d_{2}\right)$ tends to the derivative of the fractional filter $(1-L)^{-\delta}$ evaluated at $\delta=0$; that is, to the linear filter $J(L)=-\log (1-L)=\sum_{j=1}^{\infty} j^{-1} L^{j}$. In this case, the regression (7) can be rewritten as

$$
\Delta y_{t}=\phi_{2} \sum_{j=1}^{t-1} j^{-1} \Delta y_{t-j}+u_{t}
$$$$
(t=1, \ldots, T) \text {. }
$$

Interestingly, the $t$ test for the significance of $\phi_{2}$ in (9) is Robinson's LM test statistic given in (3), apart from a different, but asymptotically equivalent (un- 
der local alternatives) normalization. To see this, note that the sample covariance between the dependent and independent variable in (9) is given by $\sum_{j=1}^{T-1} j^{-1} \widehat{\gamma}_{\Delta y}(j)$, where $\widehat{\gamma}_{\Delta y}(j)$ denotes the sample autocovariance of order $j$ of $\Delta y_{t}$. The $t$ test for the significance of $\phi_{2}$ in (9) has been considered by Agiakloglou and Newbold (1994) and Breitung and Hassler (2002). Although the $t$ test based on (9) is asymptotically locally equivalent to the $t$ test based on (7), in a fixed alternative framework the $t$ test based on (7) should be preferred to the one based on (9). The reason is that there exists no value for $\phi_{2}$ that makes $u_{t}$ in (9) be both i.i.d. and independent of the regressor for fixed alternatives and, hence, the regressor $\sum_{j=1}^{t-1} j^{-1} \Delta y_{t-j}$ does not maximize the correlation with the regressand $\Delta y_{t}$.

The next theorem establishes the asymptotic properties of $t_{\phi}$, where $d_{2}$ is allowed to be stochastic with limit not necessarily equal to $d$. In particular, under local alternatives, it shows that the test is asymptotically equivalent to the optimal Robinson LM test when $d_{2}$ is optimally chosen. The proof is given in Appendix 1 of the Supplement (Lobato and Velasco (2007)). Introduce the function $h$ :

$$
h\left(d_{2}\right)=\frac{\sum_{j=1}^{\infty} j^{-1} \pi_{j}\left(d_{2}-1\right)}{\sqrt{\sum_{i=1}^{\infty} \pi_{i}\left(d_{2}-1\right)^{2}}}, \quad d_{2}>0.5, \quad d_{2} \neq 1,
$$

and $h(1)=\sqrt{\sum_{j=1}^{\infty} j^{-2}}=\sqrt{\pi^{2} / 6}$.

THEOREM 1: Under the assumption that the DGP is given by

$$
\Delta^{d} y_{t} 1\{t>0\}=\varepsilon_{t},
$$

where $\varepsilon_{t}$ is i.i.d. with finite fourth moment, the asymptotic properties of the t-test statistic $t_{\phi}$ for testing $\phi_{2}=0$ in (7), where the input $\widehat{d}_{2}$ of $z_{t-1}$ satisfies

$$
\widehat{d}_{2}=d_{2}+o_{p}\left(T^{-\tau}\right) \quad \text { with } \tau>0 \text { and } \widehat{d}_{2}>0.5 \text {, }
$$

for some fixed $d_{2}>0.5$, are given by the following statements:

(a) Under the null $(d=1)$,

$$
t_{\phi} \rightarrow{ }_{d} \mathrm{~N}(0,1) .
$$

(b) Under fixed alternatives $(d<1)$, the test based on $t_{\phi}$ is consistent.

(c) Under local alternatives $(d=1-\delta / \sqrt{T}, \delta>0)$,

$$
t_{\phi} \rightarrow{ }_{d} \mathrm{~N}\left(-\delta h\left(d_{2}\right), 1\right) .
$$

REMARK 1.1: The drift function $h$ is plotted in Figure 1. Note that $h$ achieves an absolute maximum at $d_{2}=1$ and that $h(1)$ equals the noncentrality 


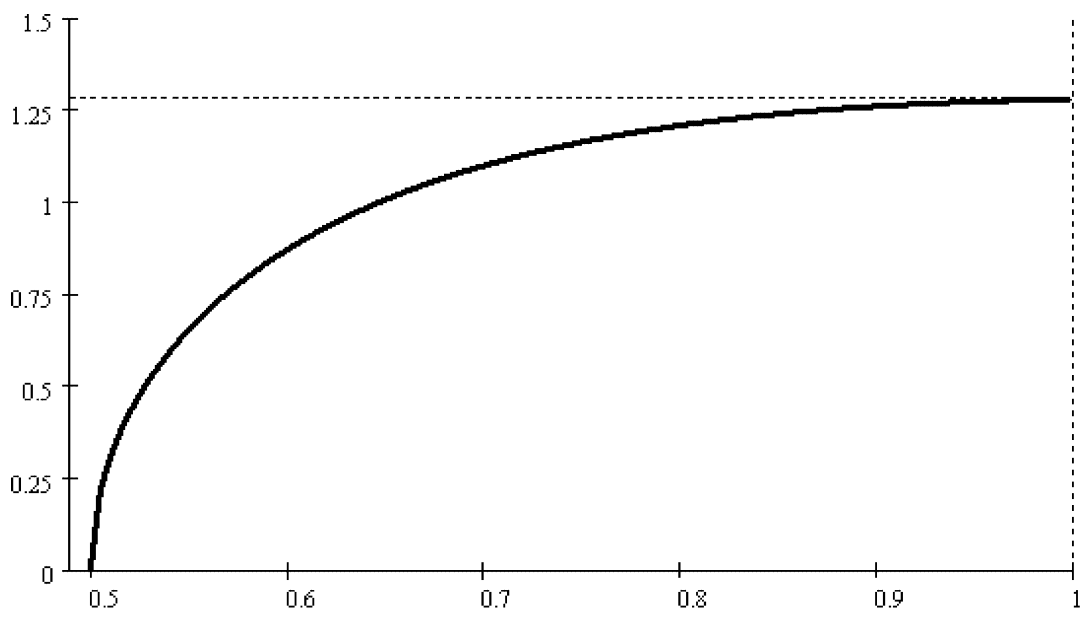

d2

FIGURE 1.-Plot of $h\left(d_{1}\right)$. The horizontal line at $\pi / \sqrt{6} \approx 1.28$ corresponds to Robinson's LM test.

parameter of the locally optimal Robinson LM test, so the new test is locally asymptotically equivalent to this test when a consistent estimator of $d$, which satisfies condition (10), is employed as the input $d_{2}$. Also note that the drift of DGM's test statistic is 1 , so the asymptotic relative efficiency of the DGM test is 0.79 .

REMARK 1.2: Notice that the first part of condition (10) holds with $d_{2}=d$ for any estimator of $d$ that is consistent at a power rate, so that not only are parametric $\sqrt{T}$-consistent estimators of $d$ as proposed by DGM (e.g., Velasco and Robinson (2000)) allowed, but also many semiparametric estimators for an appropriate choice of the bandwidth parameter can be employed, such as those of Velasco (1999a, 1999b). The condition $\widehat{d}_{2}>0.5$ can be imposed naturally for implicitly defined memory estimators, such as the Gaussian semiparametric procedure of Robinson (1995), whereas for other estimators this condition could be replaced by the condition $\left|\widehat{d}_{2}\right| \leq K$ for some $K>0$ as in Robinson and Hualde's (2003) Assumption 3. The purpose of these conditions is to guarantee that the use of estimated regressors does not alter the asymptotic distribution of the test statistic, given that $\phi_{2}=0$ under the null; see, for instance, the discussion in Wooldridge (2002, Chapter 6). 


\section{SHORT RUN DYNAMICS}

The analysis in the previous sections imposes that the DGP is $\Delta^{d} y_{t}=\varepsilon_{t}$, where $\varepsilon_{t}$ is white noise. Practically, it is more appropriate to allow for $\Delta^{d} y_{t}$ to be serially correlated. In this section we consider that the DGP of $y_{t}$ is given by the autoregressive fractionally integrated moving average $\operatorname{ARFIMA}(p, d, 0)$ model

$$
\alpha(L) \Delta^{d} y_{t} 1\{t>0\}=\varepsilon_{t}
$$$$
(t=1,2, \ldots),
$$

where $\alpha(L)=1-\alpha_{1} L-\cdots-\alpha_{p} L^{p}$ is a polynomial in the lag operator with all its roots outside the unit circle. Note that this DGP can be written as

$$
\alpha(L) \Delta y_{t}=\alpha(L)\left(1-\Delta^{d-1}\right) \Delta y_{t}+\varepsilon_{t},
$$

or, equivalently, by letting the same dependent variable be on the left as in the pure fractional case,

$$
\Delta y_{t}=\alpha(L)\left(1-\Delta^{d-1}\right) \Delta y_{t}+(1-\alpha(L)) \Delta y_{t}+\varepsilon_{t} .
$$

Note that none of the $t$ tests considered in the previous sections can properly control the type I error because of the short run correlation induced by $\alpha(L)$ on $\Delta^{d} y_{t}$. The use of an augmented test based on the $t$ statistic associated to the coefficient of the regressor $\Delta^{d_{1}} y_{t-1}$ in a regression of $\Delta y_{t}$ on $\Delta^{d_{1}} y_{t-1}$ and $p$ lags of $\Delta y_{t}$ was proposed by DGM. Similarly, to keep the linearity of the regression model, we could simplify Equation (12) by suppressing the factor $\alpha(L)$ in the first regressor, and considering the regression of $\Delta y_{t}$ on $z_{t-1}\left(d_{2}\right)$ and $p$ lags of $\Delta y_{t}$. It is simple to show that this test can properly control the type I error, but it is inefficient due to the deletion of the factor $\alpha(L)$ in the first regressor of (12). Hence, we prefer to analyze the following two-step approach that leads to efficient tests.

Note that Equation (12) motivates the nonlinear regression model

$$
\Delta y_{t}=\varphi_{2}\left\{\alpha(L)\left(\Delta^{d_{2}-1}-1\right) \Delta y_{t}\right\}+\sum_{j=1}^{p} \alpha_{j} \Delta y_{t-j}+u_{t},
$$

which is similar to (6), except for the inclusion of the lags of $\Delta y_{t}$ and for the filter $\alpha(L)$ in the regressor whose significance is tested. Similar to the white noise case, for continuity reasons, we propose to use the rescaled regression model

$$
\Delta y_{t}=\phi_{2}\left\{\alpha(L) z_{t-1}\left(d_{2}\right)\right\}+\sum_{j=1}^{p} \alpha_{j} \Delta y_{t-j}+u_{t}
$$

with $z_{t-1}\left(d_{2}\right)$ defined in (8). As in the white noise case, the DGP (12) is a particular case of model (13). Under the null hypothesis, $\Delta y_{t}-\sum_{j=1}^{p} \alpha_{j} \Delta y_{t-j}$ is i.i.d. 
and, therefore, $\phi_{2}=0$ for any value of $d_{2}$, with $u_{t}=\varepsilon_{t}$. Under the alternative hypothesis, when $d_{2}$ is chosen equal to $d, \phi_{2}=d-1$ (so that the DGP (12) is recovered), model (13) is properly specified, with regressors $\alpha(L) z_{t-1}(d)$ and $\left\{\Delta y_{t-j}\right\}_{j=1}^{p}$ independent of the i.i.d. error term $u_{t}=\varepsilon_{t}$. This is not true when $d_{2}$ is chosen differently from the true value of $d$, indicating that an appropriate selection of the input $d_{2}$ is needed to derive optimal tests.

Estimation of model (13) is complicated because of the nonlinearity in the parameters $\phi_{2}$ and $\alpha=\left(\alpha_{1}, \ldots, \alpha_{p}\right)^{\prime}$. Compared to the white noise case, note that the practical problem arises because the vector $\alpha$ is unknown and, so, the regressor $\alpha(L) z_{t-1}\left(d_{2}\right)$ is unfeasible. Hence, first we need to obtain a consistent estimate of $\alpha$. We propose the following two-step procedure.

First, estimate by OLS the equation

$$
\Delta^{\widehat{d}_{2}} y_{t}=\sum_{j=1}^{p} \alpha_{j} \Delta^{\widehat{d}_{2}} y_{t-j}+u_{t},
$$

where the input $\widehat{d}_{2}$ is any consistent estimator of $d$ that satisfies

$$
\widehat{d}_{2}=d+O_{p}\left(T^{-\tau}\right), \quad \tau>0, \quad \text { and } \quad\left|\widehat{d}_{2}\right| \leq K \quad \text { for some } K>0 .
$$

The OLS estimator of $\alpha$ is consistent with a convergence rate that depends on the convergence rate of the estimator of $d$ (see the proof of Theorem 1 that is given in Appendix 1 in the Supplement (Lobato and Velasco (2007))).

Second, estimate by OLS the equation

$$
\Delta y_{t}=\phi_{2}\left[\widehat{\alpha}(L) z_{t-1}\left(\widehat{d}_{2}\right)\right]+\sum_{j=1}^{p} \alpha_{j} \Delta y_{t-j}+v_{t},
$$

where $\widehat{\alpha}(L)$ denotes the estimator of $\alpha(L)$ from the first step and where $\widehat{d}_{2}$ takes the same value as in the first step. The asymptotic null distribution of the resulting $t$ statistic associated to $\phi_{2}$ is still the standard normal, as if $\widehat{\alpha}$ (and $\widehat{d}_{2}$ ) were fixed, because $\phi_{2}=0$ under $H_{0}$; see Remark 1.2 in Section 2. Under the alternative, because $\widehat{\alpha}$ converges to the true $\alpha$ and $\widehat{d}_{2}$ converges to the true $d$, a $t$ test for $\phi_{2}=0$ based on (16) has asymptotic properties similar to the test based on model (13) with $d_{2}=d$.

The next theorem establishes the asymptotic properties of the $t$ test statistic $t_{\phi}$ for testing $\phi_{2}=0$ in (16). The proof is given in Appendix 2 in the Supplement (Lobato and Velasco (2007)). Introduce the notation

$$
\omega^{2}=\frac{\pi^{2}}{6}-\kappa^{\prime} \Phi^{-1} \kappa
$$

$\kappa=\left(\kappa_{1}, \ldots, \kappa_{p}\right)^{\prime}$ with $\kappa_{k}=\sum_{j=k}^{\infty} j^{-1} c_{j-k}, k=1, \ldots, p$, where the $c_{j}$ are the coefficients of $L^{j}$ in the expansion of $1 / \alpha(L)$ and where $\Phi=\left[\Phi_{k, j}\right], \Phi_{k, j}=$ 
$\sum_{t=0}^{\infty} c_{t} c_{t+|k-j|}, k, j=1, \ldots, p$, denotes the Fisher information matrix for $\alpha$ under Gaussianity.

THEOREM 2: Under the assumption that the DGP is an $\operatorname{ARFIMA}(p, d, 0)$ model defined as

$$
\alpha(L) \Delta^{d} y_{t} 1\{t>0\}=\varepsilon_{t},
$$

where $\varepsilon_{t}$ is i.i.d. with finite fourth moment and $\alpha(L)=1-\alpha_{1} L-\cdots-\alpha_{p} L^{p}$ is a polynomial in the lag operator with all its roots outside the unit circle, the asymptotic properties of the $t$-ratio test statistic $t_{\phi}$ for testing $\phi_{2}=0$ in (16), where the $\widehat{\alpha}$ used in the regressor $\left\{\widehat{\alpha}(L) z_{t-1}\left(\widehat{d}_{2}\right)\right\}$ is obtained from the OLS estimation of (14) and the input $\widehat{d}_{2}$ of $z_{t-1}$ satisfies (15), are given by the following statements:

(a) Under the null $(d=1)$,

$$
t_{\phi} \rightarrow{ }_{d} \mathrm{~N}(0,1) .
$$

(b) Under fixed alternatives $(d<1)$, the test based on $t_{\phi}$ is consistent.

(c) Under local alternatives $(d=1-\delta / \sqrt{T}, \delta>0)$,

$$
t_{\phi} \rightarrow{ }_{d} \mathrm{~N}(-\delta \omega, 1)
$$

REMARK 2.1: Note that the drift of the asymptotic distribution under local alternatives coincides with that in Robinson (1994a, Theorem 4) and, so, the proposed Wald test is asymptotically locally equivalent to the optimal LM test, similarly to the white noise case. Comparing $\omega$ with $h(1)=\pi / \sqrt{6}$ given in Theorem 1, we can observe the asymptotic loss of efficiency due to the estimation of the short memory parameters.

REMARK 2.2: In Theorem 2, for simplicity, we only considered the case where consistent estimators of $d$ are employed as $\widehat{d}_{2}$, because, as in Theorem 1 , these are the only values that lead to efficient tests in this framework. Under condition (10), when $d_{2} \neq d, t$ tests are asymptotically standard normal under the null, but inefficient.

REMARK 2.3: In a framework similar to that in this section, Breitung and Hassler (2002) also proposed a two-step procedure that presents two main differences. First, it is based on the local regressor $z_{t-1}(1)$, and, second, in their first step the $\alpha$ 's are estimated consistently only under the null hypothesis. However, note that these selections for the long and short term parameters lead to a regression model where the regressor whose significance is tested does not maximize the partial correlation with $\Delta y_{t}$ given the $p$ lags of $\Delta y_{t}$ for fixed alternatives. 


\section{SIMULATIONS}

Next, we examine the finite sample performance of the considered tests by means of a small Monte Carlo study. We consider two Gaussian DGP's: a pure fractionally integrated process and an $\operatorname{ARFIMA}(1, d, 0)$. Tables I and II report the results for the first DGP for a nominal level of 0.05 and two samples sizes, 100 and 500, respectively. For Table I, the number of replications is 50,000; for Table II, it is 10,000 . The parameter $d$ takes values from 0.5 to 1 with increments of 0.05 in Table I and it takes values from 0.8 to 1 with increments of 0.025 in Table II. These tables report the results of the time domain version of Robinson's LM test, of DGM's test, and of the new efficient Wald test. Regarding DGM's test and the new efficient test, note that the reported results correspond to unfeasible implementations of the tests because they assume that the true $d$ is known and ignore the sampling error associated with the estimation of $d$. We also computed these tests using parametric and semiparametric estimators of $d$ with similar results, which are omitted for brevity, the only noticeable difference being some slight additional size distortion when $T=100$. These tables report the size results $(d=1)$ and size-adjusted power instead of raw power $(d<1)$, because Robinson's LM test is somewhat conservative compared to DGM's test and the new efficient test for $T=100$.

The main messages from these two tables are the following. First, as expected, the most powerful test is the proposed efficient test, which can improve the size-adjusted power up to $30 \%$ with respect to DGM's original proposal. Second, compared to the efficient test, the loss of power of the LM test is greater the further the alternative is from the null, reflecting the local character of this test.

In Table III we consider the case where the DGP is a Gaussian ARFIMA(1, $d, 0)$ with autoregressive parameter $\alpha_{1}=\{-0.5,0,0.3,0.6,0.8\}$. We only report the results for one negative value for $\alpha_{1}$, because for other negative values the results were similar, contrary to the $\alpha_{1}>0$ case, where finite sample power depends greatly on $\alpha_{1}$. In addition, the most empirically relevant case is when $\alpha_{1}>0$. The parameter $d$ takes values from 0.5 to 1 with increments of 0.05 . As

\section{TABLE I}

Monte Carlo Size $(d=1)$ AND (Size Adjusted) Power $(d<1)$ OF Robinson's Time Domain LM Test, DGM's Test, AND THE NEW EFFICIENT WALD TEST ${ }^{\mathrm{a}}$

\begin{tabular}{lcccccccccccc}
\hline \hline$T=100$ & $d$ & 0.5 & 0.55 & 0.6 & 0.65 & 0.7 & 0.75 & 0.8 & 0.85 & 0.9 & 0.95 & 1 \\
\hline LM & & 99.9 & 99.7 & 99.1 & 97.1 & 92.1 & 81.5 & 64.6 & 44.6 & 25.8 & 12.6 & 4.53 \\
DGM & $d_{1}=d$ & 100 & 99.9 & 99.6 & 98.2 & 93.6 & 82.1 & 64.2 & 43.0 & 24.5 & 11.9 & 5.27 \\
EFF-W & $d_{2}=d$ & 100 & 100 & 100 & 99.9 & 98.3 & 91.8 & 76.8 & 53.6 & 30.7 & 13.7 & 5.59 \\
\hline
\end{tabular}

\footnotetext{
a Percentage of rejections based on 5\% nominal level. The series follows a pure Gaussian fractionally integrated process with parameter $d$. Sample size is 100 . The number of replications is 50,000.
} 
TABLE II

Monte Carlo Size $(d=1)$ And (Size Adjusted) Power $(d<1)$ of Robinson's Time DOMAIN LM TEST, DGM's TEST, AND THE NEW EFFICIENT WALD TEST ${ }^{\mathrm{a}}$

\begin{tabular}{lcccccccccc}
\hline \hline$T=500$ & $d$ & 0.8 & 0.825 & 0.85 & 0.875 & 0.9 & 0.925 & 0.95 & 0.975 & 1 \\
\hline LM & & 100 & 99.9 & 99.1 & 95.9 & 83.9 & 62.5 & 35.8 & 15.1 & 5.50 \\
DGM & $d_{1}=d$ & 99.9 & 99.3 & 96.4 & 88.6 & 73.0 & 51.4 & 29.0 & 13.2 & 4.86 \\
EFF-W & $d_{2}=d$ & 100 & 100 & 99.7 & 97.5 & 87.9 & 66.5 & 38.8 & 16.3 & 5.12 \\
\hline
\end{tabular}

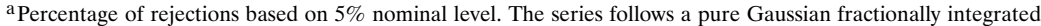
process with parameter $d$. Sample size is 500. The number of replications is 10,000 .
}

above, we use 0.05 as the nominal level, and consider two samples sizes, 100 and 500, with 50,000 and 10,000 replications, respectively.

We report results for three tests: (a) the original unfeasible augmented DGM test that uses $d_{1}=d$, (b) the unfeasible two-step efficient test that ignores the sampling variation associated with the estimation of $d$, and (c) the feasible two-step test that uses as $d_{2}$ the Gaussian semiparametric estimator of Velasco (1999b) with bandwidth $m=T^{0.55}$. In Table III these tests are denoted by ADGM, 2S, and 2SSP, respectively. For the three tests we included one lag in the augmented regression.

Next, we comment on the results from Table III. Note that under the null hypothesis, for any value of $\alpha_{1}$, the empirical rejection probabilities are above the nominal level for all tests. This size distortion is especially apparent for the feasible 2SSP test, as we would expect, because the estimation of $d$ leads to an increase in the sampling variation of the test statistic. Hence, we report sizeadjusted power instead of raw power. The most noticeable feature of Table III is that power is higher when the serial correlation is negative, and it deteriorates substantially and rapidly as $\alpha_{1}$ becomes positive and large. For instance, it is interesting to observe the enormous loss of power associated to an increase of $\alpha_{1}$ from 0.6 to 0.8 . When $\alpha_{1}=0.8$ and $T=100$, the three tests report very low size-adjusted power, indicating that, in the presence of moderate or strong positively correlated innovations, long time series are needed so as to discriminate reasonably well between fractional integration and weak dependence.

Table III also indicates that the unfeasible efficient $2 \mathrm{~S}$ test presents higher size-adjusted power than the unfeasible ADGM, as expected, and that this difference is especially relevant when positive serial correlation is present, the case of most practical interest. In particular, for $\alpha_{1}=0.8$ and $T=500$, the $2 \mathrm{~S}$ test presents twice as much power as ADGM test for values of $d$ between 0.6 and 0.7. In addition, note that the loss of power of the feasible 2SSP test compared to the unfeasible $2 \mathrm{~S}$ test is rather moderate, except for the $\alpha_{1}=0.8$ case. Also, the case $\alpha_{1}=0$ is interesting for comparing the loss of power due to introducing an irrelevant regressor in the augmented regression. Comparing Tables I and II with Table III, for the $\alpha_{1}=0$ case, it is noticeable that this loss of power is substantial, up to $50 \%$, indicating that a careful selection of the 
TABLE III

Monte Carlo Size $(d=1)$ And (Size Adjusted) Power $(d<1)$ OF the Unfeasible

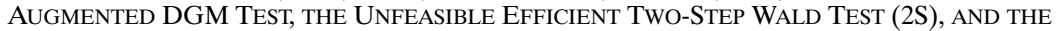
FEASIBLE TWO-STEP TEST BASED ON A SEMIPARAMETRIC ESTIMATOR OF $d$ (2SSP)a

\begin{tabular}{|c|c|c|c|c|c|c|c|c|c|c|c|c|}
\hline$\alpha_{1}$ & $d$ & 0.5 & 0.55 & 0.6 & 0.65 & 0.7 & 0.75 & 0.8 & 0.85 & 0.9 & 0.95 & 1 \\
\hline \multirow{4}{*}{-0.5} & \multicolumn{12}{|c|}{$T=100$} \\
\hline & ADGM & 100 & 99.9 & 99.3 & 97.4 & 91.1 & 78.5 & 60.1 & 40.0 & 22.8 & 11.3 & 6.81 \\
\hline & $2 \mathrm{~S}$ & 100 & 100 & 99.8 & 98.1 & 92.3 & 79.8 & 61.3 & 40.5 & 23.1 & 11.7 & 6.80 \\
\hline & 2SSP & 100 & 99.9 & 99.3 & 97.0 & 90.2 & 76.7 & 57.7 & 37.9 & 21.5 & 11.0 & 7.70 \\
\hline \multirow[t]{3}{*}{0} & ADGM & 99.4 & 97.9 & 92.7 & 84.8 & 71 & 55 & 39.7 & 26.1 & 16 & 8.9 & 6.91 \\
\hline & $2 \mathrm{~S}$ & 99.8 & 98 & 95 & & & & & & & 9.4 & 6.73 \\
\hline & 2SSP & 99.3 & 97 & 93.1 & 84.2 & 70.8 & 54.7 & 38.8 & 25.3 & 15.5 & 9.0 & 7.67 \\
\hline \multirow[t]{3}{*}{0.3} & $\mathrm{ADG}$ & 94 & 86 & 75 & 6 & 4 & & 4 & 17.3 & 11 & 7.5 & 6.87 \\
\hline & $2 S$ & 95.9 & 85 & 79.3 & 65.9 & 51.6 & 38 & 26.7 & 18.1 & 12 & 8.0 & 6.86 \\
\hline & 2SSP & 93.3 & 86.4 & 76.2 & 63.4 & 49.7 & 37.0 & 26.1 & 17.9 & 11.8 & & 7.33 \\
\hline \multirow[t]{3}{*}{0.6} & $\mathrm{ADC}$ & 56 & & & 9 & 1 & & 10.4 & 2 & & 5.5 & 7.11 \\
\hline & $2 \mathrm{~S}$ & 62 & & 3 & 2 & 21 & & & 9 & & 6.2 & 6.95 \\
\hline & 2SSP & 57.9 & 47.2 & 37.0 & 28.4 & 21.6 & 16 & 12 & 9.3 & & 6.1 & 6.79 \\
\hline \multirow[t]{4}{*}{0.8} & $\mathrm{ADC}$ & 11 & 8 & 5 & $A$ & & & 2 & 3.0 & & 3.9 & 7.36 \\
\hline & $2 \mathrm{~S}$ & 15 & 10 & 8.1 & & 5.0 & & & 3.9 & & 5 & 7.20 \\
\hline & 2SSP & 12.3 & 9.3 & 7.1 & 5.7 & 4.6 & 4.0 & 3.7 & 3. & 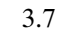 & 4.2 & 6.45 \\
\hline & \multicolumn{12}{|c|}{$T=500$} \\
\hline \multirow[t]{3}{*}{-0.5} & ADGM & 100 & 100 & 100 & 100 & 100 & 10 & 99.9 & 96.8 & 73.6 & 28.9 & 5.79 \\
\hline & $2 \mathrm{~S}$ & 100 & 100 & 100 & 100 & 100 & 10 & 100 & 97.6 & 75.0 & 29.4 & 5.74 \\
\hline & 2SSP & 100 & 100 & 100 & 100 & 100 & 100 & 99.9 & 96.9 & 73.2 & 28.7 & 6.63 \\
\hline \multirow[t]{3}{*}{0} & $\mathrm{ADG}$ & 100 & 100 & 100 & 100 & 100 & 99 & 97.6 & 83.7 & 51.2 & 19.9 & 5.73 \\
\hline & $2 \mathrm{~S}$ & 100 & & & 100 & 100 & 100 & & 86.6 & & 20.9 & 5.54 \\
\hline & 2SSP & 100 & 100 & 100 & 100 & 100 & 99.9 & 97.9 & 84.4 & 51.6 & 20.5 & 6.49 \\
\hline \multirow[t]{3}{*}{0.3} & ADGM & 100 & 100 & 100 & 100 & 99.5 & 97.3 & 84.0 & 59.2 & 32.1 & 14.2 & 5.55 \\
\hline & $2 \mathrm{~S}$ & 100 & 100 & 100 & 100 & & & 88.8 & 64.6 & & 15.6 & 5.43 \\
\hline & $2 \mathrm{SSP}$ & 100 & 100 & 100 & 100 & 99.9 & 97.7 & 86.7 & 63.0 & 34.6 & 15.2 & 6.27 \\
\hline \multirow[t]{3}{*}{0.6} & ADGM & 100 & 99 & 98. & 90.4 & 75 & 54 & 35.2 & 21.4 & 12 & 7.6 & 5.61 \\
\hline & $2 \mathrm{~S}$ & 100 & 100 & 99.8 & 96.4 & 85.2 & 65.2 & 43.8 & 26.5 & & 8.6 & 5.41 \\
\hline & 2SSP & 100 & 99.9 & 98.7 & 93.7 & 82.1 & 63.3 & 43.6 & 26.9 & 15.8 & 8.9 & 6.18 \\
\hline \multirow[t]{3}{*}{0.8} & ADGM & 63.7 & 40. & 22.8 & 12.5 & 7.0 & 4.1 & 3.0 & 2.7 & 2.8 & 3.5 & 6.01 \\
\hline & $2 \mathrm{~S}$ & & & 41. & & & & 5.8 & 4.6 & & 4.3 & 5.97 \\
\hline & $2 \mathrm{SSP}$ & 68.9 & 51.9 & 36.1 & 23.9 & 15.0 & 9.5 & 6.2 & 4.8 & 4.4 & 4.5 & 6.22 \\
\hline
\end{tabular}

${ }^{a}$ Percentage of rejections based on $5 \%$ nominal level. The series follows an ARFIMA(1, $\left.d, 0\right)$ with Gaussian errors. The autoregressive parameter is $\alpha_{1}$. The number of lags of $\Delta y_{t}$ included in the augmented regression is 1 . The number of replications is 50,000 when $T=100$ and 10,000 when $T=500$.

number of lags included in the augmented regression is crucial to balance the trade-off between size and power that a researcher faces in practice. Finally, notice that the nonmonotonic behavior for the power figures, when $\alpha_{1}=0.8$, could be due to the fact that the high persistence of the AR(1) makes it difficult 
to distinguish a unit root from long memory for high values of $d$ and relatively small sample sizes.

\section{CONCLUSIONS AND FURTHER RESEARCH}

In this article we introduced efficient Wald tests for fractional unit roots by using a model based auxiliary regression. The proposed tests are locally asymptotically equivalent to the locally optimal LM tests of Robinson (1991, 1994a). In addition, the first-order asymptotic properties of the proposed tests are not affected by the estimation of short or long memory parameters. We finish with some suggestions on further research. Because our test presents a clear analogy with the original Dickey-Fuller test, it can be interesting to study the cases where deterministic trends or structural breaks may appear in the data generating process. In addition, note that the techniques employed in this paper can also be applied in a multivariate framework for testing simply and efficiently for (fractional) cointegration. In this article, we just considered the case where the short range correlation follows an autoregressive process of known order. An extension of practical interest is to examine the robustness of these procedures in the presence of short term serial correlation of unknown form. This analysis entails studying the behavior of these procedures when the order of the autoregression increases with the sample size. Finally, studying the effects of truncating the fractional filter is another area that deserves more attention. In this respect, Robinson (2005) provided an approach for handling this issue.

Centro de Investigación Económica, Instituto Tecnológico Autónomo de México, Av. Camino Sta. Teresa 930, México D.F. 10700, Mexico; ilobato@itam.mx and

Departamento de Economía, Universidad Carlos III de Madrid, Calle Madrid 126, 28903 Getafe (Madrid), Spain; carlos.velasco@uc3m.es.

Manuscript received May, 2005; final revision received October, 2006.

\section{REFERENCES}

AgiaKloglou, C., AND P. Newbold (1994): "Lagrange Multiplier Tests for Fractional Difference," Journal of Time Series Analysis, 15, 253-262. [580]

BREITUNG, J., AND U. HASSLER (2002): "Inference on the Cointegration Rank in Fractionally Integrated Processes," Journal of Econometrics, 110, 167-185. [580,584]

DiCKEY, D. A., AND W. A. FUlleR (1979): "Distribution of Estimators of Autoregressive Time Series with a Unit Root," Journal of the American Statistical Association, 74, 427-431. [576]

Dolado, J. J., J. GONZALO, AND L. MAYORAL (2002): “A Fractional Dickey-Fuller Test for Unit Roots," Econometrica, 70, 1963-2006. [575,576]

LOBATO, I. N., AND C. VELASCO (2006): “Optimal Fractional Dickey-Fuller Tests,” Econometrics Journal, 9, 492-510. [577]

(2007): "Supplement to 'Efficient Wald Tests for Fractional Unit Roots'," Econometrica Supplementary Material, 75, http://www.econometricsociety.org/ecta/supmat/5854proofs. pdf. [580,583] 
Robinson, P. M. (1991): "Testing for Strong Serial Correlation and Dynamic Conditional Heteroskedasticity in Multiple Regression," Journal of Econometrics, 47, 67-84. [575,577,588] (1994a): "Efficient Tests of Nonstationary Hypotheses," Journal of the American Statistical Association, 89, 1420-1437. [575,577,584,588]

(1994b): "Time Series with Strong Dependence," in Advances in Econometrics: Sixth World Congress, ed. by C. A. Sims, Vol. 1. Cambridge, U.K.: Cambridge University Press, 47-95. [577]

(1995): "Gaussian Semiparametric Estimation of Long Range Dependence," The Annals of Statistics, 23, 1630-1661. [581]

(2005): "Efficiency Improvements in Inference on Stationary and Nonstationary Fractional Time Series," The Annals of Statistics, 33, 1800-1842. [588]

ROBINSON, P. M., AND J. HUALDE (2003): "Cointegration in Fractional Systems with Unknown Integration Orders," Econometrica, 71, 1727-1766. [581]

TANAKA, K. (1999): “The Nonstationary Fractional Unit Root," Econometric Theory, 15, 249-264. [577]

VELASCO, C. (1999a): “Non-Stationary Log-Periodogram Regression,” Journal of Econometrics, 91, 325-371. [581]

(1999b): "Gaussian Semiparametric Estimation of Non-Stationary Time Series," Journal of Time Series Analysis, 20, 87-127. [581,586]

VElasco, C., AND P. M. RoBINSON (2000): "Whittle Pseudo-Maximum Likelihood Estimates of Non-Stationary Time Series," Journal of the American Statistical Association, 95, 1229-1243. [581]

WoOldridge, J. M. (2002): Econometric Analysis of Cross Section and Panel Data. Cambridge, MA: MIT Press. [581] 